\title{
线粒体相关内质网膜在自噬起始中作用的 研究进展
}

\author{
刘玉姣 ${ }^{1,2}$, 陈佺 $^{2}$, 刘垒 ${ }^{1,3 *}$ \\ 1. 中国科学院动物研究所, 膜生物学国家重点实验室, 北京 100101; \\ 2. 南开大学生命科学院, 药物化学生物学国家重点实验室, 天津 300071 ; \\ 3. 北京干细胞与再生医学研究院, 北京 100101 \\ *联系人, E-mail: liulei@ioz.ac.cn \\ 收稿日期: 2021-10-18; 接受日期: 2021-12-15; 网络版发表日期: 2022-01-05
}

摘要 自噬是真核细胞自我降解自我循环以维持自身稳态的重要过程. 目前在自噬相关的分子机制研究中, 关于 自噬起始尤其是自噬膜的来源还有诸多疑点。越来越多证据表明，线粒体相关内质网膜(mitochondria-related membrane, MAM) 作为内质网和线粒体之间的通讯中心, 可能在自噬起始过程中有重要的调控功能. 本文将讨论 和总结MAM在自噬起始阶段发挥的作用，为将来的研究提供关键线索.

关键词 MAM, 自噬, 膜

自噬在进化中高度保守, 是细胞利用溶酶体消化 错误或多余组分实现物质循环再利用的生物学过程. 自噬使得细胞适应不良的生长环境, 被认为是细胞 的一种关键的生存机制 ${ }^{[1,2]}$. 自噬的底物包括错误折 叠或聚集的蛋白, 如引起阿尔茨海默症的 tau蛋白、 引起帕金森症的 $\alpha$-突触核蛋白, 受损或多余的细胞 器, 如线粒体、核糖体, 以及病原体, 如伤寒沙门氏 菌、结核分枝杆菌等 ${ }^{[3]}$. 正常情况下, 细胞会保持低 水平的基础自噬, 但是在应激情况下, 如饥饿、缺 氧、生长因子剥夺、病原体感染等, 细胞会上调自 噬水平以应对不同压力状态从而保证细胞生存 ${ }^{[4]}$. 很 多疾病的发生发展往往伴随着自噬水平的异常, 因 此自噬是很多疾病治疗的潜在靶点 ${ }^{[5]}$. 然而关于自噬 分子机制的研究, 特别是自噬起始的研究尚未完全
明晰。

人们过去认为, 不同的细胞器有各自相对独立的 生物学功能, 然而大量研究表明, 细胞器之间也存在 物理联系从而实现细胞器之间的通讯 ${ }^{[6]}$. 线粒体相关 内质网膜(mitochondria-associated membrane, MAM) 作为联接内质网和线粒体的关键位点, 介导二者以脂 质、蛋白质、代谢产物、 $\mathrm{Ca}^{2+}$ 等形式交换重要信息. MAM由内质网的子域、线粒体外膜(outer mitochondrial membrane, OMM) 和一系列蛋白质组成. MAM中 的蛋白质根据功能可以分为不同的组: 钻转运相关、 脂质代谢相关、自噬相关、胰岛素信号转导相关等. 因此MAM参与多种细胞关键事件, 具有重要的生物学 功能 ${ }^{[7 ~ 9]}$. 本文主要讨论当前MAM在自噬起始过程中 发挥的重要作用. 


\section{MAM概述}

线粒体内质网接触位点(mitochondria-endoplasmic reticulum contact sites, MERCs)指线粒体和内质网紧密 相连但膜不融合的区域, MERCs的生化对应物是线粒 体相关内质网膜，可以通过差速离心得到 ${ }^{[10,11]}$. 1956 年, Bernhard等人首次在大鼠肝脏细胞中观察到线粒 体与ER之间紧密接触，随后在多种细胞中都能找到此 类形态学证据, 直到Vance从大鼠肝脏中生化分离出这 种结构, 才将其命名为 $\mathrm{MAM}^{[12,13]}$. 电子显微镜证据表 明, ER和OMM之间的相互作用距离约为 $10 \sim 30 \mathrm{~nm}$, 该 物理维度与蛋白质桥接双膜的观点保持一致 ${ }^{[14 \sim 16]}$. 活 细胞成像显示, HeLa细胞中约 $20 \%$ 的线粒体稳定地保 持与内质网的动态链接，虽然内质网膜和线粒体都是 高度动态的细胞器, 两者接触部位会不断变化, 但 MAM结构稳定存在 ${ }^{[17,18]}$. 通过去垢剂、高浓度盐或蛋 白酶孵育粗线粒体可以分离两个细胞系, 说明MAM中 的双膜联系是可逆的 ${ }^{[12]}$. MAM的生物功能受ER和线 粒体之间接触数量、长度和厚度的严格控制.

通过对MAM蛋白质组的全面分析，在MAM中发 现了 1000 多种蛋白质，根据MAM蛋白质的定位可以 将其分成三类：（ｉ）仅定位于MAM中的蛋白质 (“MAM居留蛋白质”); (ii ) 定位于MAM但也存在于 其他细胞组分的蛋白(“MAM富集蛋白质”); (iii) 暂时 存在于MAM中的蛋白(“MAM相关蛋白”). 这些蛋白质 参与多个重要细胞生物学过程 ${ }^{[19-23]}$ (图1).

MAM的功能实现依赖于MAM结构的完整性. MAM中有些蛋白质参与维持MAM的结构稳定. 酵母 中, $\mathrm{ER}$ 和线粒体连接主要通过 $\mathrm{ER}$ 线粒体接触结构 (ER-mitochondria encounter structure, ERMES)来保证. ERMES 是一种多蛋白复合物, 包含ER针定蛋白 $M m m 1$ 、细胞质结合蛋白 $M d m 12$ 以及 $O M M$ 蛋白 Mdm34和Mdm10, 通过可溶性脂载体蛋白实现高效的 脂质运输 ${ }^{[2,25]}$. ERMES的功能涉及ER和线粒体之间的 磷脂交换、线粒体蛋白输入、线粒体DNA复制以及 协调线粒体动态, 这些功能受到ERMES复合物的调节 亚基 $\mathrm{Ca}^{2+}$ 结合rho样GTPase Gem1 的影响 ${ }^{[26-28]}$. Gem1与 哺乳动物中的Miro-2是直系同源物，与ERMES的组装 无关, 但能调节ERMES的大小和数量. Gem 1 包含四个 潜在的调控模块：两个GTPase区域作为分子开关响应 核苷酸交换因子(nucleotide exchange factors, GEFs)、
GTPase激活蛋白(GTPase-activating proteins, GAPs)和 鸟嘌呤核苷酸解离抑制剂(guanine nucleotide dissociation inhibitors, GDIs). 两个功能性EF区域响应 $\mathrm{Ca}^{2+}$ 浓 度升高 ${ }^{[29]}$.

哺乳动物细胞中, ER-线粒体连接更复杂, 可根据 功能划分为不同的蛋白质组. (i) 与线粒体动力学相 关. Miro蛋白家族(Miro1和Miro2)是线粒体运动的主 要调节因子, 可通过结合驱动蛋白, 将线粒体系在细 胞骨架上介导线粒体的运动 ${ }^{[30]}$. 位于线粒体外膜的 Fis 1 和Mff通过招募动力相关蛋白DRP 1 从而破坏 MAM的完整性，STX 17通过调节Drp1活性和定位参 与该过程 ${ }^{[31,32]} . \mathrm{Mfn} 2$ 是一种介导线粒体融合的GTP酶, $\mathrm{ER}$ 中的Mfn2与线粒体外膜上的Mfn $1 / 2$ 组装成同源或 异源二聚物，当Mfn2过表达时, ER和线粒体的相互作 用会增强 ${ }^{[33]}$. Parkin作为一种E3泛素连接酶, 可以通过 影响Mfn2的泛素化来改变MAM的完整性 ${ }^{[34]}$. (ii) 与 $\mathrm{Ca}^{2+}$ 转运相关. MAM作为ER和线粒体的桥梁, 是两个 细胞器之间的 $\mathrm{Ca}^{2+}$ 转移缓冲区. $\mathrm{IP}_{3} \mathrm{R} / \mathrm{Grp} 75 / \mathrm{VDAC}$ 是 MAM中钙离子转运的核心结构, 作为MAM的标志物, 是与ER线粒体偶联有关的最重要的蛋白质复合物. $\mathrm{IP}_{3} \mathrm{R}$ 是位于 $E R$ 中最重要的钙通道之一, 通过控制 $\mathrm{Ca}^{2+}$ 的释放影响细胞的代谢和自噬. VDAC位于线粒体外 膜, 介导线粒体对 $\mathrm{Ca}^{2+}$ 的吸收, Grp75与 $\mathrm{IP}_{3} \mathrm{R}$ 和VDAC 结合, 提高相互作用的稳定性从而提高 $\mathrm{Ca}^{2+}$ 的转移效

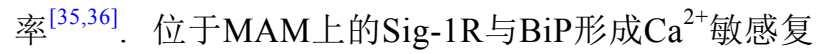
合物，通过稳定 $\mathrm{IP}_{3} \mathrm{R}$ 影响 $\mathrm{Ca}^{2+}$ 在 $\mathrm{ER}$ 和线粒体之间的转 运, 从而增加ATP的产生 ${ }^{[37]}$. PTPIP51和VAPB相互作 用，代表了 $\mathrm{ER}$ 和线粒体之间 $\mathrm{Ca}^{2+}$ 交换的另一个平台. VAPB通过C端跨膜结构域针定到ER膜，PTPIP51位于 OMM中, 是一种微管相关蛋白, 通过形成多种蛋白结 构复合物来执行不同的生物学功能. VAPB-PTPIP51复 合物被破坏, 会导致ER-线粒体接触解偶联和 $\mathrm{Ca}^{2+}$ 失 调 ${ }^{[38]}$. PDZD8在功能上与 $\mathrm{Mmm} 1$ 同源, 是哺乳动物中 的ER蛋白，对于维持MAM结构的稳定性至关重要且 对神经元中的钲离子稳态的维持发挥作用 ${ }^{[39,40]}$. 细胞 内钙稳态是细胞代谢的基础，线粒体中钘离子浓度过 低会导致细胞能量代谢紊乱, 而 $\mathrm{Ca}^{2+}$ 浓度过高会导致 细胞死亡. 正常情况下, $\mathrm{ER}$ 释放的 $\mathrm{Ca}^{2+}$ 被转运到线粒 体基质, 以激活TAC循环从而促进ATP的合成, 当过量 的 $\mathrm{Ca}^{2+}$ 被转运到线粒体时会导致线粒体渗透压调节孔 开放引起细胞调亡 ${ }^{[41]}$. (iii) 与自噬和调亡相关. Fis1和 


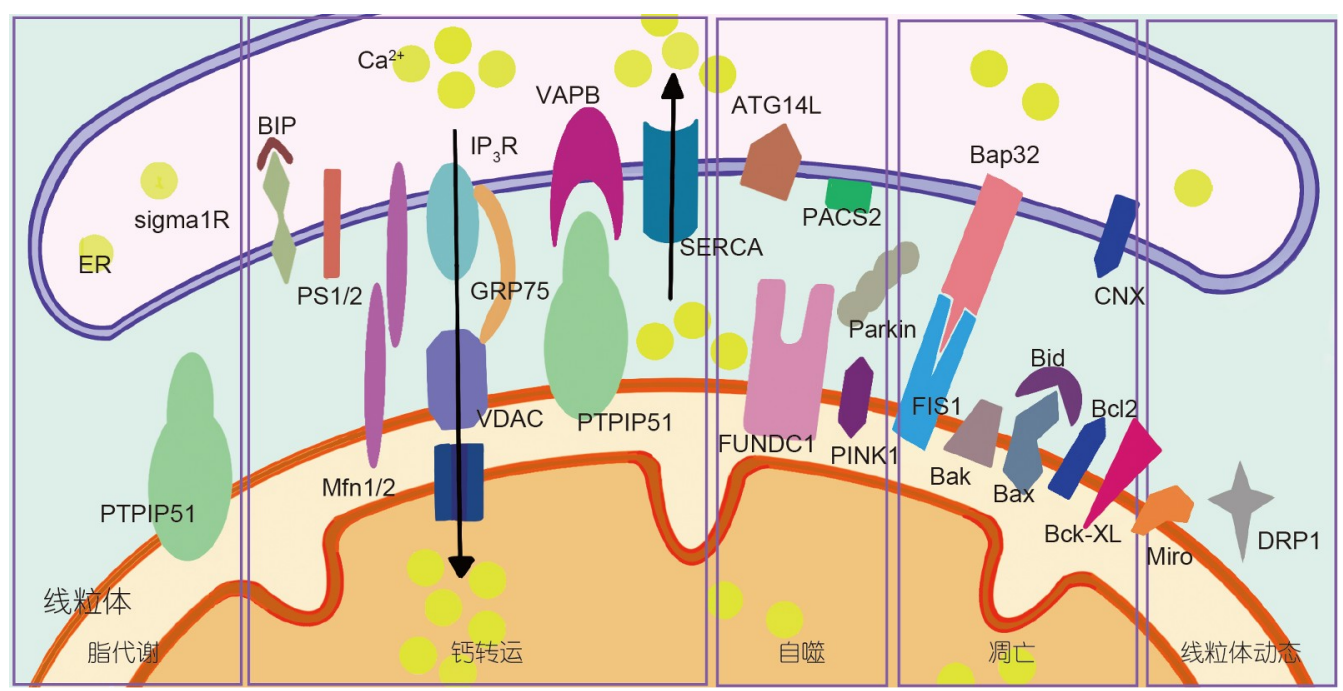

图 1 MAM蛋白质组. MAM中的蛋白质参与多个重要细胞生物学过程, 主要为调亡、线粒体动态、自噬、脂代谢和䥻转运

Figure 1 MAM proteomics. Proteins in MAM are involved in several important cellular biology processes, mainly apoptosis, mitochondrial dynamics, autophagy, lipid metabolism and calcium transport

ER上的BAP31相互作用, 参与调亡过程 ${ }^{[42]}$. PACS-2 是 MAM上的一种多功能分选蛋白，当细胞中不存在 PACS-2时，BAP31会通过caspase依赖性途径产生p20, 随后p20通过调节Drp1诱导线粒体分裂，从而破坏 MAM的完整性 ${ }^{[43]}$. BAP31和线粒体外膜的易位酶 Tom 40 形成的复合物能刺激复合物 1 的组分NDUFS 4 从细胞质转到线粒体, 从而增加线粒体复合物 1 的活性 和耗氧量 ${ }^{[44]}$. PACS-2 的过表达会增加ER-线粒体的偶 联; 降低PACS-2的表达会降低MAM的完整性并抑制 LC3-II的脂质化, 从而抑制自噬 ${ }^{[5]}$. (iv) 与脂质代谢相 关. 许多参与脂质代谢的酶都位于MAM上, MAM通过 连接内质网和线粒体参与脂质合成和运输. 磷脂酰乙 醇胺 $\mathrm{N}$-甲基转移酶(phosphatidylethanolamine Nmethyltransferase, PEMT2)与磷脂酰胆碱(phosphatidylcholine，PC)合成有关，PC通过磷脂酰丝氨酸合酶 (phosphatidylserine synthase, PSS)形成磷脂酰丝氨酸 (phosphatidylserine, PS), 再通过线粒体中的PS脱羒酶 转化为磷脂酰乙醇胺 (phosphatidyl ethanolamine, $\mathrm{PE})^{[7,46]}$. DGAT2催化三酰甘油合成并促进脂滴(lipid droplets，LDs)形成，DGAT2具有线粒体靶向信号，可 促进MAM与线粒体的关联 ${ }^{[47]}$. 上述内质网和线粒体 之间的蛋白质复合体均在维持MAM的结构完整性中 起着核心作用.

\section{MAM和自噬}

参与自噬是MAM的一个公认功能. 自噬是细胞借 助于溶酶体对不必要的或功能失调的细胞组分进行降 解的过程, 奥米伽体作为吞噬泡形成的中心是自噬起 始的最初结构, 吞噬泡不断生长将待降解物包裹形成 自噬体, 最后与溶酶体融合实现降解. 正常条件下, 细 胞发生基础水平的自噬; 在饥饿、缺氧、生长因子剥 夺等应激条件下, 自噬水平上调以平衡营养来源适应 不利生存环境 ${ }^{[1,4,48]}$.

自噬体的形成和发展涉及一系列自噬相关蛋白 (autophagy-related gene proteins, ATG蛋白)和参与膜运 输的蛋白. 自噬起始于ER中富含 3 -磷酸磷脂酰肌醇 (phosphatidylinositol-3-phosphate, PI3P)的子域, 随后 吞噬泡生长, 该过程一方面与ULK复合物和PI3激酶复 合物(VPS34, p150, BECN1, ATG14和NRBF2组成)周 期性地激活合成新脂质有关，另一方面与膜供应体 ER-高尔基中间体、高尔基体、内体、质膜等有 关 ${ }^{[49-52]}$. 吞噬泡最终成熟封闭形成自噬体的过程需要 ATG偶联系统的参与, ATG7, ATG10和ATG3介导类泛 素蛋白ATG12与ATG5相互作用, ATG12-ATG5复合物 再与ATG16相互作用介导LC3与PE的结合, 介导自噬 体的成熟 ${ }^{[53]}$. 最后, 自噬体与溶酶体融合依赖于 Rab7 及其效应体HOPS对两个细胞器的栓系, 以及SNARE 
蛋白(如STX17, SNAP29和VAMP8)介导的融合 ${ }^{[54,55]}$.

MAM在自噬中起作用从两方面的研究结果得到 支持.一方面, 对于自噬体形成至关重要的奥米伽体与 内质网相连, 一些内质网标记蛋白定位于自噬体膜; 另 一方面, 有研究表明, 线粒体在饥饿诱导的自噬中为自 噬体提供膜. Hailey等人 ${ }^{[56]}$ 发现, 线粒体定位的细胞色 素b5(具有发夹样膜针)和苂光标记的PS(可在线粒体 中转化为荧光标记的PE)在饥饿时从线粒体转移到自 噬体, 光漂白显示线粒体膜和自噬小体短暂共享. 质膜 来源的网格蛋白包被囊泡通过SNARE蛋白VAMP7及 其伴侣蛋白促进吞噬体的延伸 ${ }^{[57]}$. ATG9A阳性囊泡在 不同的细胞质区室之间循环，将膜传递到发育中的自 噬体 ${ }^{[52,58]}$.

\section{MAM和自噬启动}

关于自噬启动，目前自噬体膜的主要来源尚不清 楚，但有充分的证据表明自噬是在ER-线粒体偶联位 点开始的. 如前所述, ERMES是酵母中的ER-线粒体 偶联复合物, 通过Mmm1-Mdm12-Mdm34/Mdm10相互 作用组装, 而 Mdm34和Mdm12的泛素化是吞噬泡延伸 所必需的，这说明ER-线粒体偶联位点在酵母中参与 了自噬 ${ }^{[59]}$. 哺乳动物细胞中, 自噬前体的标志物 ATG14(静息条件下存在于胞质和ER)是PI3K复合物的 一个组成部分, 参与自噬小体的形成 ${ }^{[60]}$. 在饥饿条件 下，ATG14和DFCP1(奥米伽体标志物)的定位显著转 移到MAM，对于自噬体形成至关重要的ATG5在自噬 泡生物发生过程中易位至MAM区域，然后在自噬体 成熟后与MAM分离，而通过干扰参与ER-线粒体偶联 的蛋白质(如PACS2和MFN2)来破坏MAM时，ATG14 和DFCP1则不能正确定位在MAM中，自噬体的形成 受到抑制, 这充分说明MAM在自噬体的形成中起重 要的作用 ${ }^{[61]}$.

MAM对自噬启动的贡献与其功能息息相关. 除了 蛋白质, 脂质在自噬体的形成中也起重要作用, 特别是 磷脂和固醇. $\mathrm{PE}$ 是哺乳动物细胞中含量排名第二的磷 脂, 在自噬泡延伸过程中与自噬关键蛋白LC3结合, PS 是细胞膜结构的重要组成部分也可以与LC3结合. PE 是LC3 的主要靶标, 高水平的PE可以促进PE与LC3之 间的连接，从而促进LC3介导的自噬泡的融合和封闭。 因此，PE对于自噬体的形成可能是必不可少的 ${ }^{[62,63]}$.
此外, LDs是细胞中储存脂质的细胞器, 是合成自噬体 的重要脂质来源. 自噬过程中释放的脂肪酸(fatty acids, FAs)通过DGAT1转移到新的LDs中, 以防止FA 诱导的细胞损伤 ${ }^{[64,65]}$.

ATG2是调节自噬泡生长的关键蛋白, 它有两个亚 型, 即ATG2A和ATG2B, ATG2A能同时结合并稳定转 移数十种脂质 ${ }^{[66]}$. 自噬泡生长过程中, ATG $2 \mathrm{~A}$ 从 MAM 转移到自噬体, ATG2A通过C端一个 45 个氨基酸的结 构域针定在 MAM上, 该结构域称为 MAM定位域 (MAM localization domain, MLD), TOM40和TOM70 与MLD相互作用决定ATG2A在MAM上的定位. 此外, ATG2A与ATG9A通过其N端区域相互作用. 据此, 研究 者提出了一个模型, 即TOM40-TOM70复合物将ATG2 募集至MAM, 从而将脂质以囊泡和非囊泡的形式转移 至延伸中的自噬泡，使自噬体长大增强自噬流 ${ }^{[67]}$. 同 样，位于MAM的肿瘤抑制因子PML通过调节AMPK/ $\mathrm{mTOR} / \mathrm{ULK} 1$ 途径的活性, 影响 $\mathrm{Ca}^{2+}$ 从ER到线粒体的 转运, 从而控制自噬体的形成 ${ }^{[35,68]}$.

MAM中 $\mathrm{Ca}^{2+}$ 失调会导致自噬异常 ${ }^{[69]}$. EI24是位于 ER中参与自噬调节的蛋白, 敲除EI24会破坏MAM的 完整性并抑制原代胰腺 $\beta$ 细胞的自噬. 发生自噬时, EI24 易位至MAM并与 $\mathrm{Ca}^{2+}$ 转运核心 $\mathrm{IP}_{3} \mathrm{R}-\mathrm{Grp} 75$ $\mathrm{VDAC}$ 复合物相互作用以维持MAM结构的完整 ${ }^{[70]}$. 当 $\mathrm{ER}$ 与线粒体之间 $\mathrm{Ca}^{2+}$ 转运受到破坏时, $\mathrm{AMPK}$ 易位至 MAM并通过BECN激活自噬, 进一步说明MAM是自 噬体形成的平台 ${ }^{[71]}$. 然而, 有研究表明, 通过siRNA破 坏VAPB-PTPIP51相互作用会降低MAM的完整性, 但 这会激活自噬, 也就是说, VAPB-PTPIP51偶联通过破 坏 $\mathrm{Ca}^{2+}$ 转运来影响自噬 ${ }^{[38]}$. 这些研究表明, MAM介导 的 $\mathrm{Ca}^{2+}$ 转运与自噬之间有诸多关系.

\section{PINK/Parkin介导的线粒体自噬}

当前PINK1和Parkin途径是研究最深入的线粒体 自噬途径，与帕金森症的发展相关. 在哺乳动物细胞 中, 具有丝氨酸/苏氨酸激酶活性的PINK 1 和E3泛素连 接酶Parkin协同作用, 感知线粒体功能状态, 并通过自 噬途径标记和处理受损的线粒体 ${ }^{[72]}$. 在健康的线粒体 中, PINK1通过线粒体靶向序列被连续转运至线粒体, 并被线粒体加工肽酶(mitochondrial processing peptidase, MPP)降解，降解产物被位于线粒体内膜上的蛋 
白酶PARL裂解, 裂解后的PINK1被运回细胞质, 最终 在溶酶体中降解 ${ }^{[73]}$. 而在受损线粒体中, PINK1的切 割由于线粒体损伤而减少, 未切割的PINK1通线粒体 外膜蛋白转运酶TOM在线粒体的外膜上积累, 线粒体 丙酮酸水平也会通过促进PINK1和TOM的直接相互 作用从而影响PINK1在线粒体外膜的积累 ${ }^{[74]}$. 外膜上 聚集的PINK1同源二聚化和自磷酸后诱导Parkin改变 构象招募其易位到线粒体, 并激活Parkin的E3连接酶 活性, 随后活化的Parkin多泛素化VDAC1, p62和 SQSTM1等蛋白质, 被泛素化的底物通过LIR与LC3 结合, 从而在线粒体周围招募自噬体膜, 然后自噬体 膜进一步延伸形成成熟的自噬体，完成线粒体自 噬 ${ }^{[75,76]}$.

正常PINK/Parkin途径介导的线粒体自噬是细胞 内稳态的基础. 被泛素化的位点都会发生Parkin介导 的线粒体自噬, 而 LC3 募集的区域就位于ER和受损线 粒体之间. 此外, 在MAM中还发现了 PtdIns $3 K$ 的核心 成分BECN1, 它能增强ER和线粒体之间的连接并促进 自噬前体的形成 ${ }^{[77]}$. 因此, MAM是PINK/Parkin依赖的 线粒体自噬的起始位点. 过表达Parkin能增强MAM的 结构和功能, 促进 $\mathrm{Ca}^{2+}$ 从 $\mathrm{ER}$ 向线粒体转移, 增加线粒 体中ATP的产生 ${ }^{[34,78]}$. gp78是一种针固在ER膜上与线 粒体自噬相关的泛素连接酶(E3), 已被确认位于MAM 中. 这些证据表明, 参与PINK/Parkin介导的线粒体自 噬的核心蛋白位于MAM中, 并参与MAM完整性和功 能的调节.

\section{FUNDC1介导的线粒体自噬}

哺乳动物细胞中, FUNDC1参与受体介导的线粒 体自噬途径. FUNDC1高度保守, 包含 155 个氨基酸, 通过位于线粒体外膜的LIR区域募集LC3 并在缺氧时 启动线粒体自噬 ${ }^{[79]}$. 正常情况下, FUNDC1的 18 位酪氨 酸和13位丝氨酸分别被 $\mathrm{Src}$ 和 $\mathrm{CK} 2$ 磷酸化, 抑制其与 LC3 结合以诱导自噬, 缺氧时, 线粒体蛋白磷酸酶 PGAM5介导 13 位丝氨酸的去磷酸化, 从而使FUNDC1 与LC3结合引发线粒体自噬 ${ }^{[80]}$. 此外, ULK1作为早期 参与自噬体形成的Ser/Thr激酶, 也与FUNDC1的功能 密切相关. 缺氧或用FCCP处理时, ULK1的表达增加 并被募集到破碎的线粒体中, 转位的ULK1与FUNDC1 相互作用并促进FUNDC1上 17 位丝氨酸的磷酸化以启
动自噬 ${ }^{[81]}$. MARCH5 是可调节线粒体自噬的线粒体 $\mathrm{E} 3$ 连接酶, MARCH5与FUNDC1直接相互作用, 并通过促 进FUNDC1在119位赖氨酸的泛素化来降解FUNDC1, 并且MARCH5的存在导致FUNDC1对缺氧信号不敏 感 ${ }^{[82]}$.

关于MAM与FUNDC1介导的线粒体自噬的直接 关系, 有研究表明, FUNDC1是一种MAM定位蛋白, 与 另一种MAM蛋白 $I P_{3} R 2$ 相互作用, 介导 $I P_{3} R$ 依赖的 $\mathrm{Ca}^{2+}$ 信号从ER到线粒体和从ER到胞浆的转导 ${ }^{[83]}$. 降低 FUNDC1的表达, 细胞内 $\mathrm{Ca}^{2+}$ 水平降低, $\mathrm{Ca}^{2+}$ 敏感的 cAMP反应元件结合蛋白(cAMP-response element binding protein, CREB)会抑制Fis1的表达, 从而引起 线粒体功能障碍. 此外, FUNDC1的表达降低会破坏 $\mathrm{ER}$ 与线粒体之间的相互作用, 并降低MAM中的蛋白 质丰度 ${ }^{[84]}$. 关于MAM和FUNDC1的研究表明, 正常情 况下, MAM中存在少量FUNDC1, 而缺氧时, FUNDC1 会在MAM中大量积累 ${ }^{[85]}$. FUNDC1易位到MAM与 $\mathrm{CNX}$ 有关, $\mathrm{CNX}$ 的 $\mathrm{N}$ 末端与FUNDC1的亲水域之间存 在相互作用. 但是, CNX的N端位于ER的内腔中, 而 FUNDC 1 的亲水结构域不太可能穿透ER的内腔与 $\mathrm{CNX}$ 相互作用. 因此, 这里有一个未知的蛋白质介导 CNX和FUNDC1之间的相互作用. 在缺氧条件下, CNX耗尽可以抑制FUNDC1易位至MAM，这进一步 证实了 CNX在FUNDC1易位中的作用 ${ }^{[85]}$. 这些证据表 明, MAM为FUNDC1实现功能提供了一个平台.

还有另一种参与ER-线粒体偶联的蛋白PACS2也 在线粒体自噬中起重要作用. PACS2在动脉粥样硬化 脂质刺激的过程中介导 $\mathrm{ER}$-线粒体连接的完整性. $\mathrm{PACS} 2$ 缺失会导致MAMs的破坏和线粒体自噬体的形 成, 从而引起线粒体自噬失调 ${ }^{[86]}$.

\section{6 总结}

自噬是细胞自身稳态的重要生物学过程, 这个过 程的缺陷与许多疾病息息相关, 如神经退行性疾病、 癌症和急性肾损伤(acute kidney injury, AKI)等. 作为 $\mathrm{ER}$ 和线粒体之间的桥梁, $\mathrm{MAM}$ 在 $\mathrm{Ca}^{2+}$ 转运、脂质代 谢和自噬中发挥重要作用. 一方面, MAM是自噬相关 蛋白质实现其生物功能的平台. 另一方面, MAM的 $\mathrm{Ca}^{2+}$ 转运和脂质代谢功能会影响自噬体的生长. MAM 结构的不完整和功能缺陷会导致自噬异常. 虽然现有 
的研究已经充分证明了MAM和自噬之间的相关性, 但 仍有许多细节问题需要进一步探讨, 比如哪些蛋白质 可以调解MAM参与自噬泡的延伸? MAM与自噬异常 引起的疾病之间有没有关系? 现有支持MAM和自噬
之间关系的证据均是体外实验获得的, 还需要体内实 验进一步验证. MAM和自噬调控机制的进一步阐明, 对于MAM成为治疗自噬相关疾病的靶标具有重要 意义.

\section{参考文献}

1 Glick D, Barth S, Macleod K F. Autophagy: cellular and molecular mechanisms. J Pathol, 2010, 221: 3-12

2 Yang Y, Klionsky D J. Autophagy and disease: unanswered questions. Cell Death Differ, 2020, 27: 858-871

3 Chu C T. Mechanisms of selective autophagy and mitophagy: Implications for neurodegenerative diseases. Neurobiol Dis, 2019, 122: 23-34

4 Kim K H, Lee M S. Autophagy—a key player in cellular and body metabolism. Nat Rev Endocrinol, 2014, 10: 322-337

5 Levine B, Kroemer G. Biological functions of autophagy genes: a disease perspective. Cell, 2019, 176: 11-42

6 Bravo-Sagua R, Torrealba N, Paredes F, et al. Organelle communication: Signaling crossroads between homeostasis and disease. Int J Biochem Cell Biol, 2014, 50: 55-59

7 Vance J E. MAM (mitochondria-associated membranes) in mammalian cells: lipids and beyond. Biochim Biophys Acta, 2014, 1841: 595-609

8 Hayashi T, Rizzuto R, Hajnoczky G, et al. MAM: more than just a housekeeper. Trends Cell Biol, 2009, 19: 81-88

9 van Vliet A R, Agostinis P. Mitochondria-associated membranes and ER stress. In: Wiseman R, Haynes C, eds. Coordinating Organismal Physiology Through the Unfolded Protein Response. Current Topics in Microbiology and Immunology. Cham: Springer, 2018. 73-102

10 Ilacqua N, Sánchez-Álvarez M, Bachmann M, et al. Protein localization at mitochondria-ER contact sites in basal and stress conditions. Front Cell Dev Biol, 2017, 5

11 Herrera-Cruz M S, Simmen T. Over six decades of discovery and characterization of the architecture at mitochondria-associated membranes (MAMs). In: Tagaya M, Simmen T, eds. Organelle Contact Sites. Advances in Experimental Medicine and Biology. Singapore: Springer, 2017. $13-31$

12 Vance J E. Phospholipid synthesis in a membrane fraction associated with mitochondria. J Biol Chem, 1990, 265: 7248-7256

13 Bernhard W, Rouiller C. Close topographical relationship between mitochondria and ergastoplasm of liver cells in a definite phase of cellular activity. J Biophys Biochem Cytol, 1956, 2: 73-78

14 Csordas G, Renken C, Varnai P, et al. Structural and functional features and significance of the physical linkage between ER and mitochondria. J Cell Biol, 2006, 174: 915-921

15 Giacomello M, Pellegrini L. The coming of age of the mitochondria-ER contact: a matter of thickness. Cell Death Differ, 2016, 23: 1417-1427

16 Friedman J R, Lackner L L, West M, et al. ER tubules mark sites of mitochondrial division. Science, 2011, 334: 358-362

17 Rizzuto R, Pinton P, Carrington W, et al. Close contacts with the endoplasmic reticulum as determinants of mitochondrial Ca ${ }^{2+}$ responses. Science, 1998, 280: 1763-1766

18 Rowland A A, Voeltz G K. Endoplasmic reticulum-mitochondria contacts: function of the junction. Nat Rev Mol Cell Biol, 2012, 13: 607-615

19 Barazzuol L, Giamogante F, Calì T. Mitochondria associated membranes (MAMs): architecture and physiopathological role. Cell Calcium, 2021, 94: 102343

20 Zhang A, Williamson C D, Wong D S, et al. Quantitative proteomic analyses of human cytomegalovirus-induced restructuring of endoplasmic reticulum-mitochondrial contacts at late times of infection. Mol Cell Proteomics, 2011, 10: M111.009936

21 Poston C N, Krishnan S C, Bazemore-Walker C R. In-depth proteomic analysis of mammalian mitochondria-associated membranes (MAM). J Proteomics, 2013, 79: 219-230

22 Sala-Vila A, Navarro-Lérida I, Sánchez-Alvarez M, et al. Interplay between hepatic mitochondria-associated membranes, lipid metabolism and caveolin-1 in mice. Sci Rep, 2016, 6: 27351

23 Wang X, Wen Y, Dong J, et al. Systematic in-depth proteomic analysis of mitochondria-associated endoplasmic reticulum membranes in mouse and human testes. Proteomics, 2018, 18: 1700478

24 Lang A, John Peter A T, Kornmann B. ER-mitochondria contact sites in yeast: beyond the myths of ERMES. Curr Opin Cell Biol, 2015, 35: 7-12

25 Kawano S, Tamura Y, Kojima R, et al. Structure-function insights into direct lipid transfer between membranes by Mmm1-Mdm12 of ERMES. J 
Cell Biol, 2018, 217: 959-974

26 Kornmann B, Currie E, Collins S R, et al. An ER-mitochondria tethering complex revealed by a synthetic biology screen. Science, 2009, 325: $477-481$

27 Meeusen S, Nunnari J. Evidence for a two membrane-spanning autonomous mitochondrial DNA replisome. J Cell Biol, 2003, 163: 503-510

28 Meisinger C, Rissler M, Chacinska A, et al. The mitochondrial morphology protein Mdm10 functions in assembly of the preprotein translocase of the outer membrane. Dev Cell, 2004, 7: 61-71

29 Kornmann B, Osman C, Walter P. The conserved GTPase Gem1 regulates endoplasmic reticulum-mitochondria connections. Proc Natl Acad Sci USA, 2011, 108: 14151-14156

30 Fransson S, Ruusala A, Aspenström P. The atypical Rho GTPases Miro-1 and Miro-2 have essential roles in mitochondrial trafficking. Biochem Biophys Res Commun, 2006, 344: 500-510

31 Losón O C, Song Z, Chen H, et al. Fis1, Mff, MiD49, and MiD51 mediate Drp1 recruitment in mitochondrial fission. Mol Biol Cell, 2013, 24: $659-667$

32 Arasaki K, Shimizu H, Mogari H, et al. A role for the ancient SNARE syntaxin 17 in regulating mitochondrial division. Dev Cell, 2015, 32: 304317

33 Filadi R, Greotti E, Turacchio G, et al. Mitofusin 2 ablation increases endoplasmic reticulum-mitochondria coupling. Proc Natl Acad Sci USA, 2015, 112: E2174-E2181

34 Basso V, Marchesan E, Peggion C, et al. Regulation of ER-mitochondria contacts by Parkin via Mfn2. Pharmacol Res, 2018, 138: 43-56

35 Pedriali G, Rimessi A, Sbano L, et al. Regulation of endoplasmic reticulum-mitochondria $\mathrm{Ca}^{2+}$ transfer and its importance for anti-cancer therapies. Front Oncol, 2017, 7

36 Basso V, Marchesan E, Ziviani E. A trio has turned into a quartet: DJ-1 interacts with the IP3R-Grp75-VDAC complex to control ERmitochondria interaction. Cell Calcium, 2020, 87: 102186

37 Hayashi T, Su T P. Sigma-1 receptor chaperones at the ER-mitochondrion interface regulate $\mathrm{Ca}^{2+}$ signaling and cell survival. Cell, 2007, 131: $596-610$

38 Gomez-Suaga P, Paillusson S, Stoica R, et al. The ER-mitochondria tethering complex VAPB-PTPIP51 regulates autophagy. Curr Biol, 2017, 27: 371-385

39 Hirabayashi Y, Kwon S K, Paek H, et al. ER-mitochondria tethering by PDZD8 regulates $\mathrm{Ca}^{2+}$ dynamics in mammalian neurons. Science, 2017, 358: $623-630$

40 Elbaz-Alon Y, Guo Y, Segev N, et al. PDZD8 interacts with Protrudin and Rab7 at ER-late endosome membrane contact sites associated with mitochondria. Nat Commun, 2020, 11: 3645

41 Yu H, Sun C, Gong Q, et al. Mitochondria-associated endoplasmic reticulum membranes in breast cancer. Front Cell Dev Biol, 2021,9

42 Iwasawa R, Mahul-Mellier A L, Datler C, et al. Fis1 and Bap31 bridge the mitochondria-ER interface to establish a platform for apoptosis induction. EMBO J, 2011, 30: 556-568

43 Breckenridge D G, Stojanovic M, Marcellus R C, et al. Caspase cleavage product of BAP31 induces mitochondrial fission through endoplasmic reticulum calcium signals, enhancing cytochrome c release to the cytosol. J Cell Biol, 2003, 160: 1115-1127

44 Namba T. BAP31 regulates mitochondrial function via interaction with Tom40 within ER-mitochondria contact sites. Sci Adv, 2019, 5: eaaw1386

45 Li C, Li L, Yang M, et al. PACS-2: a key regulator of mitochondria-associated membranes (MAMs). Pharmacol Res, 2020, 160: 105080

46 Vance D E, Walkey C J, Cui Z. Phosphatidylethanolamine N-methyltransferase from liver. Biochim Biophys Acta, 1997, 1348: 142-150

47 Stone S J, Levin M C, Zhou P, et al. The endoplasmic reticulum enzyme DGAT2 is found in mitochondria-associated membranes and has a mitochondrial targeting signal that promotes its association with mitochondria. J Biol Chem, 2009, 284: 5352-5361

48 Parzych K R, Klionsky D J. An overview of autophagy: morphology, mechanism, and regulation. Antioxid Redox Signal, 2014, 20: 460-473

49 Axe E L, Walker S A, Manifava M, et al. Autophagosome formation from membrane compartments enriched in phosphatidylinositol 3-phosphate and dynamically connected to the endoplasmic reticulum. J Cell Biol, 2008, 182: 685-701

50 Melia T J, Lystad A H, Simonsen A. Autophagosome biogenesis: From membrane growth to closure. J Cell Biol, 2020,219

51 Shima T, Kirisako H, Nakatogawa H. COPII vesicles contribute to autophagosomal membranes. J Cell Biol, 2019, 218: 1503-1510

52 De Tito S, Hervás J H, van Vliet A R, et al. The Golgi as an assembly line to the autophagosome. Trends Biochem Sci, 2020, 45: 484-496 
53 Wesselborg S, Stork B. Autophagy signal transduction by ATG proteins: from hierarchies to networks. Cell Mol Life Sci, 2015, 72: 4721-4757

54 Itakura E, Kishi-Itakura C, Mizushima N. The hairpin-type tail-anchored SNARE syntaxin 17 targets to autophagosomes for fusion with endosomes/lysosomes. Cell, 2012, 151: 1256-1269

55 Jiang P, Nishimura T, Sakamaki Y, et al. The HOPS complex mediates autophagosome-lysosome fusion through interaction with syntaxin 17. Mol Biol Cell, 2014, 25: 1327-1337

56 Hailey D W, Rambold A S, Satpute-Krishnan P, et al. Mitochondria supply membranes for autophagosome biogenesis during starvation. Cell, 2010, 141: 656-667

57 Ravikumar B, Moreau K, Jahreiss L, et al. Plasma membrane contributes to the formation of pre-autophagosomal structures. Nat Cell Biol, 2010, 12: $747-757$

58 Søreng K, Munson M J, Lamb C A, et al. SNX18 regulates ATG9A trafficking from recycling endosomes by recruiting Dynamin-2. EMBO Rep, 2018, 19

59 Belgareh-Touzé N, Cavellini L, Cohen M M. Ubiquitination of ERMES components by the E3 ligase Rsp5 is involved in mitophagy. Autophagy, 2017, 13: 114-132

60 Diao J, Liu R, Rong Y, et al. ATG14 promotes membrane tethering and fusion of autophagosomes to endolysosomes. Nature, 2015, 520: 563-566

61 Hamasaki M, Furuta N, Matsuda A, et al. Autophagosomes form at ER-mitochondria contact sites. Nature, 2013, 495: 389-393

62 Sou Y, Tanida I, Komatsu M, et al. Phosphatidylserine in addition to phosphatidylethanolamine is an in vitro target of the mammalian Atg8 modifiers, LC3, GABARAP, and GATE-16. J Biol Chem, 2006, 281: 3017-3024

63 Nair U, Yen W L, Mari M, et al. A role for Atg8-PE deconjugation in autophagosome biogenesis. Autophagy, 2012, 8: 780-793

64 Shpilka T, Welter E, Borovsky N, et al. Lipid droplets and their component triglycerides and steryl esters regulate autophagosome biogenesis. EMBO J, 2015, 34: 2117-2131

65 Nguyen T B, Louie S M, Daniele J R, et al. DGAT1-dependent lipid droplet biogenesis protects mitochondrial function during starvation-induced autophagy. Dev Cell, 2017, 42: 9-21.e5

66 Valverde D P, Yu S, Boggavarapu V, et al. ATG2 transports lipids to promote autophagosome biogenesis. J Cell Biol, 2019, 218: 1787-1798

67 Tang Z, Takahashi Y, Wang H G. ATG2 regulation of phagophore expansion at mitochondria-associated ER membranes. Autophagy, 2019, 15: $2165-2166$

68 Missiroli S, Bonora M, Patergnani S, et al. PML at mitochondria-associated membranes is critical for the repression of autophagy and cancer development. Cell Rep, 2016, 16: 2415-2427

69 Hu Y X, Han X S, Jing Q. $\mathrm{Ca}^{2+}$ ion and autophagy. Adv Exp Med Biol, 2019, 1206: 151-166

70 Yuan L, Liu Q, Wang Z, et al. Correction to: EI24 tethers endoplasmic reticulum and mitochondria to regulate autophagy flux. Cell Mol Life Sci, 2020, 77: 2255-2256

71 Ahumada-Castro U, Silva-Pavez E, Lovy A, et al. MTOR-independent autophagy induced by interrupted endoplasmic reticulum-mitochondrial $\mathrm{Ca}^{2+}$ communication: a dead end in cancer cells. Autophagy, 2019, 15: 358-361

72 Eiyama A, Okamoto K. PINK1/Parkin-mediated mitophagy in mammalian cells. Curr Opin Cell Biol, 2015, 33: 95-101

73 Greene A W, Grenier K, Aguileta M A, et al. Mitochondrial processing peptidase regulates PINK1 processing, import and Parkin recruitment. EMBO Rep, 2012, 13: 378-385

74 Park S, Choi S G, Yoo S M, et al. Pyruvate stimulates mitophagy via PINK1 stabilization. Cell Signal, 2015, 27: 1824-1830

75 Rasool S, Soya N, Truong L, et al. PINK1 autophosphorylation is required for ubiquitin recognition. EMBO Rep, 2018, 19: e44981

76 Geisler S, Holmström K M, Skujat D, et al. PINK1/Parkin-mediated mitophagy is dependent on VDAC1 and p62/SQSTM1. Nat Cell Biol, 2010, 12: $119-131$

77 Gelmetti V, De Rosa P, Torosantucci L, et al. PINK1 and BECN1 relocalize at mitochondria-associated membranes during mitophagy and promote ER-mitochondria tethering and autophagosome formation. Autophagy, 2017, 13: 654-669

78 Calì T, Ottolini D, Negro A, et al. Enhanced Parkin levels favor ER-mitochondria crosstalk and guarantee $\mathrm{Ca}^{2+}$ transfer to sustain cell bioenergetics. Biochim Biophys Acta, 2013, 1832: 495-508

79 Liu L, Feng D, Chen G, et al. Mitochondrial outer-membrane protein FUNDC1 mediates hypoxia-induced mitophagy in mammalian cells. Nat Cell Biol, 2012, 14: 177-185

80 Chen G, Han Z, Feng D, et al. A regulatory signaling loop comprising the PGAM5 phosphatase and CK2 controls receptor-mediated mitophagy. 
Mol Cell, 2014, 54: 362-377

81 Wu W, Tian W, Hu Z, et al. ULK1 translocates to mitochondria and phosphorylates FUNDC1 to regulate mitophagy. EMBO Rep, 2014, 15: 566575

82 Chen Z, Liu L, Cheng Q, et al. Mitochondrial E3 ligase MARCH5 regulates FUNDC1 to fine-tune hypoxic mitophagy. EMBO Rep, 2017, 18: 495-509

83 Wang C, Dai X, Wu S, et al. FUNDC1-dependent mitochondria-associated endoplasmic reticulum membranes are involved in angiogenesis and neoangiogenesis. Nat Commun, 2021, 12: 2616

84 Wu S, Lu Q, Wang Q, et al. Binding of FUN14 domain containing 1 with inositol 1,4,5-trisphosphate receptor in mitochondria-associated endoplasmic reticulum membranes maintains mitochondrial dynamics and function in hearts in vivo. Circulation, 2017, 136: 2248-2266

$85 \mathrm{Wu} \mathrm{W}, \mathrm{Li} \mathrm{W}$, Chen $\mathrm{H}$, et al. FUNDC1 is a novel mitochondrial-associated-membrane (MAM) protein required for hypoxia-induced mitochondrial fission and mitophagy. Autophagy, 2016, 12: 1675-1676

86 Yu S, Zhang L, Liu C, et al. PACS2 is required for ox-LDL-induced endothelial cell apoptosis by regulating mitochondria-associated ER membrane formation and mitochondrial $\mathrm{Ca}^{2+}$ elevation. Exp Cell Res, 2019, 379: 191-202

\title{
The role of mitochondria-associated membranes in autophagy initiation
}

\author{
LIU YuJiao ${ }^{1,2}$, CHEN Quan ${ }^{2} \&$ LIU Lei ${ }^{1,3}$ \\ 1 State Key Laboratory of Membrane Biology, Institute of Zoology, Chinese Academy of Sciences, Beijing 100101, China; \\ 2 State Key Laboratory of Pharmaceutical Chemical Biology, College of Life Sciences, Nankai University, Tianjin 300071, China; \\ 3 Beijing Institute of Stem Cell and Regenerative Medicine, Beijing 100101, China
}

Autophagy is an important process in eukaryotic cells to self-degrade and self-recycle in order to maintain homeostasis. At present, at the study of the molecular mechanisms related to autophagy there are many doubts about the initiation of autophagy, especially the source of autophagy membrane. Increasing evidence suggests that mitochondria-related membrane (MAM), as the communication center between the endoplasmic reticulum and mitochondria, may play an important regulatory role in the initiation of autophagy. In this paper, we will discuss and summarize the role of MAM in the initiation of autophagy, and provide key clues for future research.

\section{MAM, autophagy, membrane}

doi: $10.1360 /$ SSV-2021-0371 